\title{
La idea de Universidad y la Teología
}

\section{The Idea of University and Theology}

\section{José Ignacio MURILLO}

Universidad de Navarra. Facultad de Filosofía y Letras

Pamplona. España

ID ORCID 0000-0001-9789-5573

jimurillo@unav.es
Resumen: El lugar que tiene la teología dentro de la universidad depende del modo como se conciba la propia universidad. La universidad es una comunidad de personas empeñadas en el incremento del saber, buscado por sí mismo, y en su difusión en las diversas situaciones históricas. La libertad para llevar a cabo esta misión debe hacerse compatible con los condicionamientos que impone el contexto social en que se desarrolla. Además, la empresa del saber, que en nuestros días se ha diversificado y especializado, obliga a repensar la manera de no perder la unidad del saber. La búsqueda vigorosa de esa unidad está precisamente en el origen a la universidad. En esta empresa la teología ocupa un lugar destacado, pero al mismo tiempo discutido.

Palabras clave: Teología, Universidad, Interdisciplinariedad.
Abstract: The place occupied by theology within a university depends on how university itself is conceived. University is a community of people striving to acquire knowledge as a good in itself and spread it in different historical periods. The freedom to accomplish this mission can be rendered compatible with the social circumstances in which it is exercised. Moreover, facing with today's diversified and specified knowledge, it is necessary to look for ways of conserving knowledge's unity. The zealous quest for this unity was present in the university's origin. In that undertaking, theology played a significant yet, at the same time, debated role.

Keywords: Theology, University, Interdisciplinarity. 


\section{¿Tiene SENTIDO PREgUNTARSE POR LA IDEA DE LA UNIVERSIDAD?}

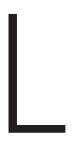

a universidad es una de las instituciones que más ha dado que pensar a lo largo de la historia. Este hecho puede que tenga una explicación sencilla. conscientes de la importancia de comprender la realidad. Es lógico, por otra parte, que esta actitud se dirija de un modo particular hacia aquello que resulta más cercano. Las crisis y los cambios afectan a todas las instituciones, pero en la universidad se viven de un modo más intenso porque es más reflexivo. Como la historia no se puede detener, es lógico que esta situación resulta casi permanente. La universidad es una cuestión abierta casi desde sus orígenes, desde el momento en que adquirió conciencia de sí misma como una realidad distinta y bien diferenciada.

Se me ha encomendado ofrecer una reflexión sobre la «idea» de la universidad en el contexto de la pregunta por el lugar que ocupa la teología en ella. Esto presupone obviamente que existe tal idea y que es relevante para comprender qué es esa institución que a lo largo del tiempo ha sido denominada universidad. Se puede decir, por tanto, que los organizadores de este congreso no estaban animados por un espíritu nominalista, pues parecían aceptar ambos supuestos. En el caso de que tengan razón, preguntarse por la idea que da sentido a aquella institución que a lo largo del tiempo ha sido llamada universidad es un buen inicio para un congreso acerca de la relación entre Teología y Universidad.

Podemos arriesgarnos a afirmar que la teología científica comparte origen con la Universidad. La aparición de ésta supuso un decisivo impulso para que el saber acerca de Dios adoptara una forma rigurosa y sistemática, cultivada por una verdadera comunidad de estudiosos. Por otra parte, en algunas de las primeras universidades, como París, Oxford y Cambridge, esta disciplina ocupó un lugar central. De este modo, la teología no sólo ha estado en la universidad desde la época en que esta última se estaba constituyendo, hace algo más de ocho siglos, sino que ha contribuido decisivamente a configurarla y conformarla. Este hecho induce a pensar que el destino de ambas se puede encontrar entrelazado.

Una vez enunciada la empresa, cabe, desde luego, preguntarse si soy la persona adecuada para responderla, pues no soy un teólogo, sino un filósofo. A propósito, me viene a la memoria la respuesta de Leonardo Polo cuando, con ocasión de una entrevista para el libro conmemorativo de los cincuenta años de 
la Facultad de Filosofía y Letras de la Universidad de Navarra, le preguntamos por la relación entre filosofía y teología: «Me considero un hombre que, gracias a Dios, tiene inteligencia y tiene fe, filosofía y teología. Para qué las voy a separar, o usar una sin la otra, cuando tengo las dos. El hecho de que una sea un saber natural y la otra sobrenatural me resulta muy poco útil en la práctica, porque yo tengo ambas. Me parecería un error no usarlas». A lo que añadía: «Bajo la actitud contraria se esconde a menudo el escepticismo, al cual siempre he tenido un gran temor» ${ }^{1}$. A mi modo de ver, una separación demasiado estricta entre el pensamiento filosófico y el teológico es un error y por eso, entre otras cosas, no me siento para nada incómodo interviniendo en un foro de teólogos.

Se puede alegar que por universidad se han entendido cosas distintas y que, por lo tanto, se trata de un concepto equívoco; pero también se puede sostener, y así lo voy a hacer en lo sucesivo, que sí existe una idea de universidad, aunque sus realizaciones hayan sido diversas a lo largo del tiempo. Esa diversidad puede depender de las circunstancias y del contexto en que esa idea se ha tenido que realizar, por una parte, y, por otra, de la intensidad con que han creído en ella quienes en cada momento la han encarnado. Si optamos por negar que la universidad responda a una idea, es evidente que no merece la pena preguntarse por la idea de la universidad y conviene más bien entender la universidad tan sólo como lo que es hoy y ahora. Pero, en mi opinión, la idea es importante, especialmente en las instituciones humanas, pues éstas se realizan y orientan en virtud de las ideas que de ellas concebimos. Por eso voy a intentar formular una idea de universidad que sea capaz de dar sentido y coherencia al empeño histórico que ha animado esta institución a lo largo de los siglos. Sólo después podremos discutir si es la que han tenido en sus mentes quienes la han realizado o si, al menos, es lo que de hecho, sabiéndolo o no, han contribuido a realizar.

Se podrá objetar que caben otras ideas de universidad distintas de la que voy a proponer, y seguramente habrá quien sostendrá que la suya es más adecuada que la mía para el tiempo que vivimos. Pero esto no vuelve vano el empeño. Lo decisivo en este caso no es tanto que la idea sea verdadera, en el sentido de que refleje exactamente los hechos, sino más bien que se defienda por sí misma, es decir, porque lo que presenta aparece como algo que merece la pena realizar y promover. El valor de esta idea se manifestará, por tanto, tam-

\footnotetext{
${ }^{1}$ Polo, L., «La investigación como fundamento de la enseñanza» (entrevista elaborada por Rubén Díez y José Ignacio Murillo que reproduce varias conversaciones con el autor), en NAVARRO, J. (ed.), Filosofía y Letras. Cincuenta años, Eunsa: Pamplona, 2006, 35.
} 
bién en su capacidad inspiradora. Así pues, en resumidas cuentas, propongo una idea de universidad que intenta dar razón del pasado, pero que, ante todo, pretende servir como inspiración. Y la presento junto a las demás para que de la comparación aparezca aquella que es preferible. En último extremo, de la idea que escojamos, de aquella que convoque nuestras aspiraciones más íntimas, dependerá el modelo de universidad que somos capaces de crear.

\section{LA REALIZACIÓN DE LA IDEA DE LA UNIVERSIDAD EN LAS DIVERSAS CIRCUNSTANCIAS HISTÓRICAS}

La universidad es una institución orientada al incremento integral del saber y a su difusión. De este modo lo característico de la universidad es convocar a una comunidad de investigadores - personas que estudian y que buscan incrementar el saber-, pero que no lo cultivan en comunidades aisladas, como en algunas escuelas de la Antigüedad, simplemente destinadas a los iniciados, sino que consideran que el saber debe ser puesto a disposición de todos. Ya los griegos habían propuesto que la búsqueda del saber elevaba la vida humana sobre el resto de los animales y nos asemejaba a la Divinidad, y que, por esta razón, era la instancia que merecía ser cultivada con más ahínco y desde la que había que organizar la propia vida. La novedad que introduce la idea de universidad es que ahora el crecimiento del saber se considera también decisivo para la sociedad en su conjunto, un vector esencial de su mejora y crecimiento. Por eso el empeño investigador de la universidad se continúa naturalmente en la difusión del saber, de tal modo que la búsqueda del saber se convierte en servicio al bien común.

Es evidente que la comunicación del saber es una condición necesaria para que éste crezca: es preciso aprender lo que se sabe y compartir los propios logros con otros investigadores. Pero la comunicación a la que aspira la universidad no se detiene en una comunidad de investigadores: el saber también debe ser comunicado a la sociedad en su conjunto. Y la primera forma de hacerlo no es aquella que tiene por soporte la piedra o el papel, sino a aquellos que se acercan a esta institución para participar de su vida. La forma más propia de comunicar el saber es escribirlo en las almas ${ }^{2}$. Por eso la comunidad

2 Éste es el sugerente título, plenamente inspirado en la tradición socrática, del libro homenaje dedicado a un gran profesor universitario: Herrero, M., Escribir en las almas: estudios en honor del prof. Rafael Alvira, Eunsa: Pamplona, 2014. 
universitaria está esencialmente constituida por profesores y alumnos. Pero además, se encuentra abierta a la totalidad del saber puesto que no cabe resignarse a compartimentar totalmente el saber sin que, al hacerlo, se pierda la tensión que conduce a buscarlo por sí mismo.

Si nos tomamos en serio esta idea de universidad, tal vez concluyamos que su existencia es lo más parecido a un milagro. Como el milagro, la universidad es un acontecimiento inesperado si tenemos en cuenta las leyes de la naturaleza. $Y$ es que lo habitual es que el saber -una rasgo inseparable de la vida humana- se busque y se ponga al servicio de los intereses prácticos. El hombre necesita saber mucho acerca de sí mismo y de lo que lo rodea tan sólo para cumplir el sencillo objetivo de sobrevivir, y mucho más todavía para poder vivir bien, para alcanzar una vida digna y propiamente humana. Sin embargo, la universidad se inspira en la tensión del ser humano hacia el saber en sí mismo y lo intenta desarrollar aun al margen de cualquier utilidad práctica inmediata. Parte de la convicción de que conocer, saber, merece la pena por sí mismo, y de que cultivar el saber es una manera de mejorar al ser humano, algo a lo que el ser humano, si aspira a la excelencia, no puede en modo alguno renunciar.

Esa tensión hacia el saber por sí mismo parece sacar a la universidad del entramado de los intereses inmediatos, y precisamente por eso la realización de la universidad en medio de un mundo configurado en gran medida por esos intereses experimenta el desafío de realizarse y dar cumplimiento a aquella misión que le da sentido en diversas circunstancias y contextos históricos.

Lo que hoy conocemos como universidad surge a finales del Medioevo, cuando el poder se concentraba en los nobles, los reyes y la Iglesia. Que el milagro se produzca en esta época no es seguramente una casualidad, pero ya desde este primer momento la novedad aparece condicionada por las circunstancias de su tiempo. Por poner un ejemplo, la relación entre profesores y alumnos parece reflejar la distinción entre el clero y los simples fieles. De hecho, el clero se identifica con aquellos que saben, algo que en las lenguas modernas todavía permanece cuando en francés se denomina irónicamente a los intelectuales les clercs o en inglés, cuando una persona que no es especialista en alguna materia, se califica a sí mismo como un laico: a layman.

En cualquier caso, la universidad, para realizar sus fines, necesita un espacio de libertad. Ese ámbito le era concedido por la Iglesia o por el rey, pero, en cualquier caso, esa libertad le resultaba imprescindible para poder organizarse en función de sus fines. En palabras del Fundador de la Universidad de 
Navarra, «La Universidad, como corporación, ha de tener la independencia de un órgano en un cuerpo vivo: libertad, dentro de su tarea específica en favor del bien común». Y se detenía a detallar algunos aspectos de esa libertad: «Algunas manifestaciones, para la efectiva realización de esta autonomía, pueden ser: libertad de elección del profesorado y de los administradores; libertad para establecer los planes de estudio; posibilidad de formar su patrimonio y de administrarlo. En una palabra, todas las condiciones necesarias para que la Universidad goce de vida propia. Teniendo esta vida propia, sabrá darla, en bien de la sociedad entera ${ }^{3}$. Puesto que la libertad no es algo que uno pueda limitarse tan sólo a esperar, conquistar ese ámbito de libertad es una de las tareas primaria del gobierno de la Universidad. Por el contrario, la debilidad de esa lucha por la libertad, el conformismo, sería un síntoma claro de que la vida de la universidad languidece.

Desde entonces, la universidad se ha debido realizar en circunstancias distintas. La aparición del Estado Moderno ha influido decisivamente en ella y ésta ha tenido que definirse respecto de esta nueva forma de concentrar y gestionar el poder. Así, por ejemplo, la idea del Estado y su organización ha contribuido a configura una nueva idea del profesor. El profesor aparece ahora como un funcionario, un servidor público cuya función consiste en proporcionar el saber a los estudiantes y que cumple en la sociedad la misión de prepararlos para ejercer una profesión. Mientras que en el Antiguo Régimen se confiaba a la universidad como misión principal formar clérigos o funcionarios reales, la misión que la legitima ahora socialmente es formar profesionales que mantengan en funcionamiento una sociedad supervisada por el Estado, que se presenta como el garante del bien público.

En este contexto, no pocas veces la libertad universitaria se ha visto amenazada. La idea de que el prototipo de la universidad es la universidad pública o, al menos, la que se encuentra respaldada y acreditada por el Estado, con frecuencia ha condicionado y entorpecido en muchos países los fines de la universidad. Además la idea moderna de universidad, cuando ha tendido a configurarse como institución pública, ha provocado otros efectos, como el hecho de que el Estado se haya arrogado el derecho de legitimar el saber que se imparte en ella. Con frecuencia, el Estado aspira a convertirse en garante de la universidad, hasta el punto de que ya no es la confianza en la institución uni-

${ }^{3}$ Escrivá de Balaguer, J., Conversaciones con Mons. Escrivá de Balaguer, Rialp: Madrid, 2001, n. 79. 
versitaria -el lugar donde se encuentran en principio los que saben-, la que refrenda lo que en ella se ofrece, sino el Estado, que respalda, por ejemplo, los títulos que en ella se imparten.

A pesar de todas las dificultades, esta situación es compatible con la realización de la idea de universidad, y de hecho en ella la universidad ha sido también capaz de dar buenos frutos. Pero la condición para que la realización de la idea de universidad en un contexto problemático no sea un mero azar o quede a merced del heroísmo o la idiosincrasia de sus protagonistas es que tanto quienes trabajan en ella $-\mathrm{y}$ no me refiero sólo a los profesores, pues son necesarias muchas personas para hacerla posible-como, especialmente, quienes la gobiernan no pierdan de vista los peligros que la situación en que desarrollan su actividad comporta. Por lo demás, la universidad se ha realizado siempre en instituciones que siguen modelos diversos: públicas y privadas, de investigación o más centradas en la docencia, colegios universitarios, etc. Pienso que la existencia de muchos tipos de universidad es beneficiosa, aunque sólo sea porque la existencia de diversidad facilita la selección natural de los modelos que son mejores o más viables, mientras que, si optáramos por un modelo único, su inviabilidad y fracaso comportaría necesariamente el fin de la institución universitaria.

En la actualidad, la situación ha cambiado respecto de la de hace algunos decenios. El papel del Estado, aunque sigue estando bien presente, ya no es tan determinante como antes. $\mathrm{Y}$ es que el Estado ha perdido su lugar como la más alta e indiscutida instancia de poder para pasar a convertirse en uno más de los agentes que lo ejercen en un contexto plural, profundamente determinado por las fuerzas de un mercado globalizado y las del capitalismo de última generación. Ahora la existencia de la universidad ya no se legitima sólo por unos títulos que dan fe de la formación que han recibido quienes pasan por sus aulas, sino que se le exige que ofrezca al mercado laboral aquello que éste le demanda.

En estas circunstancias, la libertad de la universidad para organizarse ya no depende sólo del espacio de autonomía que le proporciona el Estado, sino ante todo de su viabilidad económica, y esto la asemeja cada vez más a una empresa que tiene que velar por su productividad, si no para alcanzar el lucro, al menos para garantizar su supervivencia y la posibilidad de realizar nuevos proyectos. Esta dinámica empresarial resulta cada vez más presente en la universidad actual, en la que nos encontramos ante una situación configurada por el entrecruzamiento de las instancias burocráticas estatales -o paraestatales-, de un lado, y, de otro, por los intereses económicos y las fuerzas del mercado. 
Como en la Edad Media y en todos los periodos posteriores, el destino de la universidad depende nuevamente, de un modo especial -y ahora más acuciante- de que quienes la gobiernan tengan bien presente la idea de la universidad y, al mismo tiempo, conozcan la situación en que ésta debe ser llevada a cabo. Esto implica una invitación a los gestores que intervienen en el funcionamiento de la universidad para que comprendan bien la naturaleza de lo que tienen entre manos, y no intenten aplicar a ella modelos extraídos de otros ámbitos empresariales. Ciertamente si se pierde de vista la idea, la institución corre el peligro de convertirse en algo distinto de aquello que estaba llamada a ser. Pero también es cierto que, si se pierde de vista el contexto en que hay que llevarla a cabo, las decisiones que se toman, por bien intencionadas que sean, corren el riesgo de no lograr sus objetivos: pueden, incluso, generar efectos perversos y crear una dependencia cada vez mayor del ambiente externo, que acaba por hacer imposibles los ideales que se dicen profesar.

\section{PLURIVERSIDAD, INTERDISCIPLINARIEDAD Y UNIDAD DEL SABER:}

EL LUGAR DE LA FILOSOFÍA EN LA UNIVERSIDAD

Me he referido a las dificultades y desafíos que plantea cada una de las situaciones en que la idea de Universidad debe llevarse a cabo. Pero también resulta decisivo tener en cuenta el contexto que deriva del modo en que se ejerce en cada momento de la historia la propia tarea universitaria. Si, como hemos sostenido, la universidad aspira al incremento del saber, el modo en que éste se configura y se cultiva en cada momento influye de modo decisivo en la forma en que la actividad universitaria se lleva a cabo.

En los últimos siglos la empresa del saber se ha multiplicado en una variedad de disciplinas científicas, que son cultivadas por comunidades o grupos distintos entre los que resulta difícil la comunicación. Esta situación, con todas sus ventajas en cuanto a la extensión y rigor del saber adquirido, pone en serio riesgo la unidad del saber, sin la cual éste pierde vitalidad y vigencia en aras nuevamente de la mera aplicación práctica.

No pocos han visto este problema. Una de las soluciones que se aventura es la de unir las ciencias naturales con las humanas y sociales desde la perspectiva, supuestamente más consolidada y científica, de las primeras. Desde luego, cada vez encontramos más problemas humanos abordados, y aparentemente resueltos, con métodos científicos, y es una realidad que con mucha fre- 
cuencia la unidad del saber se busca desde los logros de las ciencias y se presenta en ensayos divulgativos más que en trabajos propiamente académicos.

La dificultad de que en este contexto se ofrezca una idea unificada del saber y también, por qué no decirlo, la exigencia de resolver problemas prácticos que exigen la colaboración de disciplinas distintas han llevado a proponer el lema de la interdisciplinariedad. Si reconducimos el problema a la idea de universidad, como el saber no existe por sí mismo, sino que es cultivado por seres humanos y sólo está vivo en ellos, la única realización adecuada de una interdisciplinariedad orientada al crecimiento del saber, consiste en el diálogo entre quienes cultivan las distintas porciones del saber. Es en ese diálogo donde, en mi opinión, cobra una importancia decisiva lo que yo entiendo por filosofía.

La filosofía no es otra cosa que el empeño incondicionado del hombre por saber. El objeto de su búsqueda no se circunscribe a ninguna región del conocimiento ni a un conjunto de preguntas bien delimitadas. Tampoco sus logros se encuentran constreñidos por las condiciones de validez que establece una determinada comunidad científica ${ }^{4}$. Por eso le corresponde también -es más, de modo especial- tratar las cuestiones últimas, las más decisivas y trascendentes, aun a pesar de su dificultad. En la Antigüedad esto resultaba claro porque por filosofía se comprendía la entera empresa del conocimiento, pero la Edad Moderna, con el desarrollo de las ciencias particulares, comenzando por la mecánica, lo ha ido troceando en disciplinas con objetivos bien delimitados, cuyo método parece ajeno a la simple búsqueda de la intelección que caracteriza a la empresa filosófica y resulta más apto para obtener un rendimiento de su aplicación práctica 5 .

Pero, al dar por hecho que los diversos ámbitos del saber eran convertidos progresivamente en disciplinas pretendidamente autónomas, la filosofía ha acabado reduciendo su objeto o bien a las cuestiones epistemológicas o a determinados ámbitos que serían puros sobre los que el método científico (¿cuál de ellos?, cabe preguntar) nada tendría que decir. Probablemente nadie es más responsable de esta retirada de la filosofía que Kant, un pensador que arroja sobre la filosofía académica una sombra alargada, hasta el punto de que podría-

\footnotetext{
${ }^{4}$ Cfr. Murillo, J. I., «¿Puede ser la filosofía científica y académica? El lugar de la filosofía en la república del saber y en la sociedad», en OrTIZ DE LANDÁZuRI, M. y GONZÁLEZ-AYESTA, C., La filosofía hoy: en la academia y en la vida, Eunsa: Pamplona, 2016, 87-104.

5 Cfr. «Son realmente autónomas las ciencias?», en Aranguren, J., Borobia, J. y LluCh, M., Fe y Razón. I Simposio Internacional Fe cristiana y cultura contemporánea, Eunsa: Pamplona, 1999, 473-480.
} 
mos reconocer su influjo en muchas corrientes de pensamiento que han asumido una actitud semejante, como la filosofía analítica, el neotomismo o la fenomenología. En cualquier caso, el resultado es la dificultad de encontrar una forma de cultivar el saber que pueda dar cumplida cuenta de la tarea de integrar los diversos fragmentos del conocimiento humano. «Lo que es válido para la historia y la física en las universidades americanas -señala MacIntyre- lo es también para la teología y la filosofía. Han llegado a ser también disciplinas casi exclusivamente especializadas y profesionalizadas. ¿En quiénes recae, entonces, en dichas universidades la tarea de integrar las diversas disciplinas, de considerar la relación de unas con otras y de preguntarse cómo contribuye cada una a la comprensión global de la naturaleza y del orden de las cosas? La respuesta es: "En nadie" ${ }^{6}$.

¿Existe una solución a este problema? Algunos autores, como el mismo MacIntyre, proponen una reforma de la universidad católica que tenga de nuevo como quicio el pensamiento filosófico y teológico. Pero, en mi opinión, esto sólo se puede lograr, y aún extender más allá de los muros de las instituciones universitarias cristianas, si se consigue involucrar en ella a investigadores de todos los campos del saber. La filosofía del futuro será fruto de un diálogo entre filósofos y científicos o será irrelevante. Esto presupone, en mi opinión, que los científicos reconozcan que su búsqueda de conocimiento, cualquiera que sea la disciplina que cultivan, es ya filosofía. Este descubrimiento conduce a reconocer los límites de la disciplina que se cultiva, pero no debe quedarse ahí. Los especialistas en la filosofía no pueden quedarse solos en la empresa de buscar y unificar el saber: requieren la colaboración de investigadores de todas las áreas. Por eso la filosofía del futuro, la que puede articular una nueva forma de entender la universidad, debería ser el fruto de un diálogo entre científicos y filósofos. Si a esto le llamamos un diálogo interdisciplinar (no me importa mucho el nombre), la universidad del futuro será interdisciplinar o, sencillamente, no será.

Nada puede legitimar la existencia de la universidad salvo la búsqueda de la verdad; y, si su motor último es la búsqueda del saber, no puede renunciar a ninguno de ellos: el saber es el saber allá donde se encuentre. Es ésta una consecuencia de la idea de la universidad para nuestro tiempo. Por otra parte, si esta tarea es importante y la universidad se incapacita para cumplir-

${ }^{6}$ MacIntyre, A., Dios, filosofía, universidades: una bistoria selectiva de la tradición filosófica católica), Nuevo Inicio: Granada, 2012, 35. 
la, será preciso encontrar otro lugar - ¿habrá alguno mejor? - dónde se pueda llevar a cabo. Romano Guardini expresa la situación con su singular clarividencia: «Cuando en el ámbito universitario, que está esencialmente determinado por el ethos de la verdad, decae la capacidad de percibir la verdad y de atenerse a la verdad conocida, ¿en qué otro lugar habrá de encontrarse entonces la verdad?» Y concluye que, en tal caso, habrá que mantener que «el centro de las decisiones determinantes para la historia se traslade a otro sitio, y que las universidades se transformen en meros centro de formación profesional» ${ }^{7}$.

Esta tensión esforzada y rigurosa hacia el saber sin limitaciones es lo que convierte a la universidad en un ámbito formativo especial e insustituible. Pero para que los investigadores y estudiantes, y, por ende, la sociedad en su conjunto, reciban ese influjo, es preciso que filósofos y científicos trabajen codo con codo en elaborar y contrastar una visión coherente de la realidad.

\section{DESAFÍOS DE LA TEOLOGÍA EN LA UNIVERSIDAD ACTUAL}

Para un cristiano, esa tarea resulta más acuciante, pero también más esperanzada. Para éste el saber culmina en la teología, en la que la búsqueda de la verdad se encuentra con la iniciativa de un Dios personal que se revela. «La teología es la hazaña intelectual más asombrosa y de mayor alcance de la historia $\gg^{8}$. Se trata de una empresa especialmente importante para la universidad, pues la fe que la anima refuerza y otorga un sentido más alto a la empresa universitaria. Pero la teología depende del mismo empeño por conocer que da lugar a la ciencia y a la filosofía y, por lo tanto, no puede elaborarse al margen de aquéllas.

Es claro que la teología se puede cultivar con fines diversos. Pero para que tenga un lugar en la universidad es preciso que los teólogos mantengan una activa conversación con científicos y filósofos. De lo contrario, la teología no se encontrará en condiciones de aspirar a los fines que le corresponden como ciencia, o, si se quiere decir de otro modo, como parte y parte privilegiada del saber humano. Ahora bien, en mi opinión, para llevar a cabo esta tarea la filosofía debe superar algunas dificultades y cumplir algunos requisitos.

\footnotetext{
${ }^{7}$ GUARDINI, R., «La responsabilidad del estudiante con la cultura», en Tres escritos sobre la universidad (edición y traducción de SáncheZ-Migallón, S.), Eunsa: Pamplona, 2012, 51-52.

${ }^{8}$ Polo, L., Filosofía y economía, OC 25, Eunsa: Pamplona, 237.
} 
La primera dificultad de la teología actual para cumplir su misión es que se encuentra en general filosóficamente -es decir, intelectualmente- empobrecida. Esto explica por qué le resulta tan difícil dar respuesta a las preguntas que le plantean las ciencias y la filosofía contemporáneas, y probablemente también que las que ofrece se encuentren condenadas a una aparente irrelevancia.

Este empobrecimiento se puede entender como fruto de una doble reacción. La primera, contra una escolástica que a menudo parecía incapaz de establecer un diálogo con el pensamiento contemporáneo; y, la segunda, frente al fracaso de algunos intentos, quizá precipitados y un poco acríticos, para actualizar las categorías filosóficas o para usar planteamientos modernos y contemporáneos con el fin de sustituir las categorías de la filosofía clásica.

El resultado de estos traumas es una tendencia a evitar las cuestiones ontológicas y epistemológicas y el diálogo con las ciencias que se traduce, o bien en el intento de buscar la racionalidad de la fe tan sólo en su coherencia interna, o bien en refugiarse en los aspectos históricos positivos para concederle un estatuto científicamente respetable. En consecuencia la teología de hoy -me refiero a la buena- es, con frecuencia, quizá más espiritual que la de antaño, pero también menos sistemática y metafísica; y, al mismo tiempo, cuando se pretende llevar a cabo una teología científica, se cae paradójicamente en un pensamiento bien poco espiritual, que adopta un método demasiado semejante al de la ciencia positiva o histórico-crítica.

Esta huida de la filosofía -o, lo que es casi lo mismo, el recurso ingenuo y meramente instrumental a ella- comporta el riesgo de cerrar sobre sí mismo el discurso de la fe y de hacerlo difícilmente inteligible para quienes se aproximan a él desde fuera. Dialogar con la filosofía y la ciencia y hacerse inteligible para quienes las cultivan no implica renunciar a nociones básicas como la de gracia, pecado, creación, alma, por poner algunos ejemplos, ni al lenguaje tradicional de la fe. Pero si renunciamos a las ciencias y a la filosofía, renunciamos también a mostrar la relación que tienen estas nociones cristianas con la experiencia y el saber humano, es decir, a que aparezcan, al menos, como posibilidades reales que pueden ponerse a lado de todo aquello que nos aparece como real.

Es, desde luego, importante que la teología sirva a la predicación de la fe y que no se separe de la actitud orante, pero esto no puede hacerse al precio de evitar confrontarla con el saber sobre el que la sociedad en la que el cristiano vive busca fundarse. Por otra parte, si la teología no es capaz de integrar 
el discurso científico y filosófico, se corre el riesgo de romper la unidad intelectual del cristiano y de que quede incapacitado para extraer consecuencias para su saber y su actividad de la fe que profesa.

A esta situación de desconexión entre la teología y los demás campos del saber contribuye decisivamente la transformación de la teología en una especialidad académica más. En el conjunto de la universidad, la teología, como la filosofía, no debe ser presentada tan sólo como una especialidad o un conjunto de especialidades académicas que corresponden sólo a un círculo de especialistas, sino que debe estar presente en todos los investigadores cristianos. Es más, puesto que la teología es, como la filosofía, fruto de una aspiración universal a la verdad, y considera desde la perspectiva de la revelación la entera realidad, hay partes importantes de la teología que deben ser desarrolladas por especialistas de otras disciplinas o, al menos, no sin contar con ellos. Esto tiene que ver con el problema de la interdisciplinariedad en la investigación teológica, tan importante en ella, por lo menos, como en la filosofía.

Pero la mediación entre la ciencia y la teología corresponde a la filosofía. Y la filosofía corre el riesgo de no ser adecuada a esta tarea si renuncia a los temas decisivos o busca asimilarse demasiado a las ciencias positivas. De hecho es esto lo que ha ocurrido con frecuencia. La filosofía se ha cerrado sistemática y acientíficamente, tomando esta actitud como un postulado inamovible, a la fe y a la teología, para convertirse a continuación en una disciplina cerrada sobre sí misma y profundamente problemática.

Como decía, la filosofía es interna a la actividad de los científicos y es deseable que sean muchos los que, de entre ellos, la desarrollen personalmente, de modo que tengan conciencia del límite de sus puntos de vista y puedan dialogar con otras ciencias, ofreciendo sus descubrimientos de tal modo que puedan ser entendidos por otros e integrados con los que provienen de otros saberes. Pero, cuando se trata de investigadores cristianos, también lo es la teología.

Otra dificultad tiene que ve con la idea que se tiene de la función de la teología y de su lugar en la sociedad civil y en la iglesia. En este sentido, la situación de la teología en la universidad no es idéntica en todas partes. Entre los lugares donde se cultiva con más intensidad, se puede distinguir, por ejemplo, el ámbito alemán, el anglosajón y los países del sur de Europa. Lo que diré a continuación vale sobre todo para estos últimos, que son el caso que mejor conozco y el que me afecta más de cerca. 
Según el antiguo esquema de la formación profesional, el cultivo de la teología en la universidad católica se legitima con frecuencia por su papel en la formación de los clérigos. Exigir los estudios de teología para los sacerdotes ha sido un gran avance. Pero esto no puede equivaler, como de hecho suele ocurrir, a aceptar que la teología sólo compete a los clérigos. En mi opinión, es preciso repensar la situación de monopolio de la teología por parte de los clérigos. Es lógico que ellos se dediquen a la teología, pero no lo es que se llegue a pensar, incluso por parte de los mismos laicos, que la teología no corresponde a quienes no lo son. Repito que no estoy hablando del hecho de que los laicos y las laicas puedan o deban aprender y enseñar teología para ocupar funciones y puestos que hoy ocupan los clérigos, sino de que la cultiven con el fin de cumplir adecuadamente los papeles que les corresponden, especialmente en el ámbito intelectual y universitario.

Éstas son algunas de las dificultades de la teología. En cualquier caso, el requisito para que ésta encuentre un lugar, es más, el lugar que le corresponde, en la universidad, más allá incluso de las instituciones cristianas que intentan realizar la idea de la universidad, es que sea capaz de iluminar eficazmente la realidad y de proporcionar verdaderas respuestas. En último extremo, y aunque sé que es ésta una afirmación no exenta de polémica, pienso que es cierto lo que señala Polo: «La visión cristiana es la única que reclama una teología. Ningún otro hecho religioso eleva hasta tal punto la capacidad intelectual del hombre» ${ }^{9}$. Y esa elevación se manifiesta en el incremento de inteligibilidad que el desarrollo de una teología ambiciosa proporciona. La capacidad de la teología para llevarlo a cabo explica que la universidad haya nacido con ella y también que, para estar completa, la universidad reclame que la teología ocupe en ella decididamente el lugar que le corresponde.

Éste es el objetivo. Otra cosa es descubrir cuáles son los medios para conseguirlo. Éstos, como la realización de la idea de universidad a lo largo de los siglos, dependen del contexto. Por eso pienso que no debemos sentirnos condicionados por las fórmulas del pasado. Sea como fuere, cabe preguntarse si la irrelevancia intelectual del pensamiento cristiano no se debe a la omisión de la tarea que nos corresponde a los cristianos. Quizá no hemos dado la importancia que le corresponde a la tarea intelectual y, cuando lo hemos hecho, hemos mimetizado modelos supuestamente exitosos, pero que no expresan la vi-

${ }^{9}$ Polo, L., Filosofía y economía, OC 25, Eunsa: Pamplona, 237. 
sión cristiana ni sirven para desarrollarla. En cuanto a la formación teológica necesaria para llevar a cabo este objetivo, sería un gran bien encontrar nuevas fórmulas para que la formación teológica, ofrecida dentro o fuera de los muros de la universidad civil, no se restrinja a algunos grupos de personas, sino que se generalice a todos los que la precisan. Para cumplir este objetivo, será preciso que el conocimiento teológico se ofrezca en una forma ordenada y sistemática, aunque esto no tienen por qué implicar que se encuentre atada a las materias tradicionales. Pero, ante todo, deberá ser, en la medida de lo posible, personalizada, para que resulte adecuada a las necesidades de cada investigador y de cada cristiano. 


\section{Bibliografía}

Aranguren, J., Borobia, J. y Lluch, M., Fe y Razón. I Simposio Internacional Fe cristiana y cultura contemporánea, Eunsa: Pamplona, 1999.

Escrivá de Balaguer, J., Conversaciones con Mons. Escrivá de Balaguer, Rialp: Madrid, 2001.

GUARDINI, R., «La responsabilidad del estudiante con la cultura», en Tres escritos sobre la universidad (edición y traducción de SáNCHEZ-MigalLón, S.), Eunsa: Pamplona, 2012.

Herrero, M., Escribir en las almas: estudios en honor del prof. Rafael Alvira, Eunsa: Pamplona, 2014.

MacInTyre, A., Dios, filosofía, universidades: una historia selectiva de la tradición filosófica católica), Nuevo Inicio: Granada, 2012.

MuRILlO, J. I., «¿Puede ser la filosofía científica y académica? El lugar de la filosofía en la república del saber y en la sociedad», en ORTIZ DE LANDÁZURI, M. y GONZÁLEZ-AYESTA, C., La filosofía hoy: en la academia y en la vida, Eunsa: Pamplona, 2016.

PolO, L., «La investigación como fundamento de la enseñanza», en NAVARRO, J. (ed.), Filosofía y Letras. Cincuenta años, Eunsa: Pamplona, 2006.

Polo, L., Filosofía y economía, OC 25, Eunsa: Pamplona. 